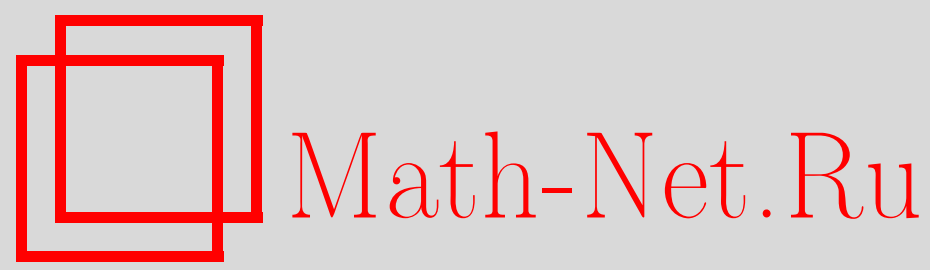

Н. А. Корешков, Картановские подалгебры с энгелевым разложением, Матем. заметки, 2002, том 72, выпуск 4, 638-640

DOI: https://doi.org/10.4213/mzm672

Использование Общероссийского математического портала Math-Net.Ru подразумевает, что вы прочитали и согласны с пользовательским соглашением http://www . mathnet.ru/rus/agreement

Параметры загрузки:

IP : 54.197 .217 .227

26 апреля 2023 г., $17: 35: 12$

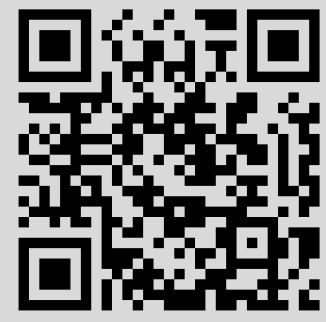




\section{КАРТАНОВСКИЕ ПОДАЛГЕБРЫ С ЭНГЕЛЕВЫМ РАЗЛОЖЕНИЕМ}

\section{Н. А. Корешков}

Как известно, в алгебре Ли $L$ над полем нулевой характеристики размерности картановских подалгебр совпадают и равны рангу этой алгебры. Более того, для любых двух картановских подалгебр $H_{1}$ и $H_{2}$ существует инвариантный автоморфизм $\varphi$ алгебры $L$ такой, что $\varphi H_{1}=H_{2}$. Для алгебр Ли над полями положительной характеристики оба утверждения неверны. Поэтому в теории модулярных алгебр Ли обычно рассматриваются картановские подалгебры с некоторыми дополнительными условиями. Например, для $p$-алгебр Ли эти условия связаны с тороидальной частью картановской подалгебры. В частности, в [1] доказано, что размерность подалгебр Картана с максимальной тороидальной частью инвариантна, а в [2] - что все такие подалгебры Картана можно получить из некоторой фиксированной подалгебры с помощью конечной цепочки элементарных преобразований, близких по форме к экспонентам от корневых внутренних дифференцирований.

В данной работе предлагается другой тип ограничений на картановскую подалгебру. А именно, для любого элемента $x$ из корневого подпространства $L_{\alpha}, \alpha \neq 0$, предполагается нильпотентность оператора $\operatorname{ad} x$. В этом случае также можно доказать инвариантность размерности картановских подалгебр, представление такой подалгебры в виде фиттинговой нулькомпоненты $C(h)$, регулярного элемента $h$ и для двух таких картановских подалгебр $H_{1}$ и $H_{2}$ существование биекции $\psi$, похожей по форме на произведение экспонент от $\operatorname{ad} x, x \in L_{\alpha}, \alpha \neq 0$, такой, что $\psi h_{1}=h_{2}$, где $H_{1}=C\left(h_{1}\right), H_{2}=C\left(h_{2}\right)$.

Перейдем к точным определениям. Пусть $H$ - картановская подалгебра в алгебре Ли $L$, а $L=H \oplus \sum_{\alpha \neq 0} L_{\alpha}$ - соответствуюшее картановское разложение. Если для любого $x \in L_{\alpha}$, $\alpha \neq 0$, оператор ad $x$ нильпотентен, то будем говорить, что $H$ - картановская подалгебра $c$ әнгелевым разложсением. Обозначим через $C(x), x \in L$, фиттингову нулькомпоненту элемента $x$, т.е.

$$
C(x)=\left\{y \in L: \operatorname{ad}^{m} x(y)=0\right\} .
$$

Элемент $x$ называется регулярным, если размерность $C(x)$ минимальна. Эта размерность называется рангом алгебры $L$.

Теорема. Пусть $L-$ алгебра Ли над алгебраически замкнутым полем $K, H$ - ее картановская подалгебра с энгелевым разложением. Тогда

1) $\operatorname{dim} H=r$, где $r-$ ранг алгебры $L$,

2) $H=C(x)$, где $x-$ регулярный әлемент,

3) для любых двух картановских подалгебр $H_{1}=C\left(h_{1}\right), H_{2}=C\left(h_{2}\right)$ с энгелевым разложсением существует биекиия

$$
\psi=E+\sum_{i_{1}+\cdots+i_{k}>0} A_{i_{1}, \ldots, i_{k}} \operatorname{ad}^{i_{1}} y_{1} \cdots \operatorname{ad}^{i_{k}} y_{k}, A_{i_{1}, \ldots, i_{k}} \in K, \quad y_{j} \in L_{\alpha}\left(H_{i}\right), \quad i=1,2,
$$

әде только конечное число коэффичиентов $A_{i_{1}, \ldots, i_{k}}$ отлично от нуля, такая, что $\psi h_{1}=h_{2}$.

ДоКАЗАТЕЛЬСТво. Для доказательства теоремы достаточно установить, что картановская подалгебра $H$ с энгелевым разложением содержит регулярньй элемент. Тогда, используя теорему о том, что фиттингова нулькомпонента регулярного элемента является подалгеброй Картана (см. например, [3]), получим два первых пункта сформулированной теоремы.

Зафиксируем базис $e_{1}, \ldots, e_{r}, e_{r+1}, \ldots, e_{n}$ алгебры $L$, согласованный с картановским разложением $L=H \oplus \sum_{\alpha \neq 0} L_{\alpha}$, причем $e_{1}, \ldots, e_{r}$ базис $H$. Для любого $x=\sum_{i=1}^{n} x_{i} e_{i}$ определим полиномиальное отображение $P: L \rightarrow L$, действующее по правилу

$$
P(x)=\left(1+x_{r+1} \text { ad } e_{r+1}\right) \cdots\left(1+x_{n} \text { ad } e_{n}\right)\left(\sum_{i=1}^{r} x_{i} e_{i}\right)=y_{1} e_{1}+\cdots+y_{n} e_{n},
$$


где $y_{i}=y_{i}\left(x_{1}, \ldots, x_{n}\right)$ - некоторые полиномиальные функции от $x_{1}, \ldots, x_{n}$.

В силу бесконечности поля $K$ существует $h_{0} \in H$ такой, что $\alpha\left(h_{0}\right) \beta\left(h_{0}\right) \cdots \gamma\left(h_{0}\right) \neq 0$ для всех ненулевых корней $\alpha, \beta, \ldots, \gamma$. Поэтому ad $h_{0}$ - невырожденное преобразование на $\bigoplus_{\alpha \neq 0} L_{\alpha}$. Повторяя рассуждения из [3], получим, что дифференциал $d_{h_{0}} P$ отображения $P$ является эпиморфизмом. Тогда в силу [3, глава IX, лемма 1] следует, что гомоморфизм $\sigma_{P}$ кольца полиномиальных функций $K[L]$ является изоморфизмом. Поэтому полиномиальное отображение $P$ - биекция [4], причем обратное к нему также полиномиально.

Кроме того, в силу эпиморфности дифференциала $d_{a} P$ для некоторого $a \in L$ имеем, что для любой полиномиальной функции $f$ на $L$ существует полиномиальная функция $g$ на $L$ такая, что $P U \supset V$, где $U=\{u \in L: f(u) \neq 0\}, V=\{v \in L: g(v) \neq 0\}$ [3, глава IX, теорема 1].

Заметим, что для любого элемента $x=\sum_{i=1}^{n} x_{i} e_{i} \in L$ характеристический многочлен $\operatorname{det}(\lambda E-\operatorname{ad} x)$ имеет вид $\lambda^{n}+\tau_{n-1} \lambda^{n-1}+\cdots+\tau_{r} \lambda^{r}$, где $\tau_{i}(x)$ - однородные многочлены степени $i$ относительно $x_{1}, \ldots, x_{n}$ и $\tau_{r} \neq 0$, но $\tau_{r-s}=0, s>0$. Сопоставляя это с вышеприведенным определением ранга алгебры $L$, видим, что он равен $r$, когда $x$ регулярен.

Пусть $f=\tau_{r} P$. Тогда для любого элемента $y$ из $V$ существует $x \in U$ такой, что $y=P x$. Следовательно, $\tau_{r}(y)=f(x) \neq 0$, т.е. $V \subset \widetilde{V}=\left\{\tilde{v} \in L: \tau_{r}(\tilde{v}) \neq 0\right\}$ - множество всех регулярных элементов из $L$.

Для элемента $y_{0} \in V$ существует элемент $x_{0} \in U$ такой, что $x_{0}=P^{-1} y_{0}$, где

$$
x_{0}=\sum_{i=1}^{n} x_{i}^{0} e_{i}, \quad x_{i}^{0}=x_{i}^{0}\left(y_{1}^{0}, \ldots, y_{n}^{0}\right),
$$

- полином от $y_{1}^{0}, \ldots, y_{n}^{0}$. Пусть $y_{0}$ - фиксированный элемент, тогда отображение

$$
\varphi_{y_{0}}=\prod_{j=r+1}^{n}\left(1+x_{j}^{0} \operatorname{ad} e_{j}\right)
$$

полиномиально на $L$ и у него существует обратное, так как каждый оператор $\operatorname{ad} e_{j}$ нильпотентен. Причем $\varphi_{y_{0}}^{-1}$ также полиномиально.

Обозначим $f_{1}=\tau_{r} \varphi_{y_{0}}^{-1}$, a $V_{1}=\left\{v_{1} \in L: f_{1}\left(v_{1}\right) \neq 0\right\}$. Если $y_{0} \in V_{1}$, то

$$
\tau_{r}\left(h_{0}\right)=\tau_{r} \varphi_{y_{0}}^{-1}\left(\varphi_{y_{0}} h_{0}\right)=f_{1}\left(y_{0}\right) \neq 0
$$

где $h_{0}=\sum_{i=1}^{r} x_{i}^{0} e_{i}-$ компонента элемента $x_{0}$, лежащая в $H$, а

$$
y_{0}=P x_{0}=\prod_{j=r+1}^{n}\left(1+x_{j}^{0} \operatorname{ad} e_{j}\right)\left(\sum_{i=1}^{r} x_{i}^{0} e_{i}\right)=\varphi y_{0} h_{0} .
$$

То есть $h_{0}$ - регулярный элемент.

Если $y_{0} \notin V_{1}$, то рассмотрим $\bar{V}_{1}=V_{1} \cap V \neq \varnothing$. В силу нашего предположения $\bar{V}_{1} \varsubsetneqq V$. Заметим, что $\bar{V}_{1}=\left\{\bar{v}_{1} \in L:\left(f_{1} g\right)\left(\bar{v}_{1}\right) \neq 0\right\}$. Возьмем $y_{1} \in \bar{V}_{1}$. Для него существует $x_{1}$ такой, что $x_{1}=P^{-1} y_{1}$, причем $y_{1}=\varphi_{y_{1}} h_{1}$, где

$$
\varphi_{y_{1}}=\prod_{j=r+1}^{n}\left(1+x_{j}^{(1)} \mathrm{ad} e_{j}\right)
$$

a $h_{1}$ - компонента $x_{1}$, лежащая в $H$.

Как выше, рассмотрим $f_{2}=\tau_{r} \varphi_{y_{1}}^{-1}$ и

$$
V_{2}=\left\{v_{2} \in L: f_{2}\left(v_{2}\right) \neq 0\right\} .
$$

Если $y_{1} \in V_{2}$, то $f_{2}\left(y_{1}\right)=\tau_{r} \varphi_{y_{1}}^{-1}\left(y_{1}\right)=\tau_{r} \varphi_{y_{1}}^{-1}\left(\varphi_{y_{1}} h_{1}\right)=\tau_{r}\left(h_{1}\right) \neq 0$. То есть $h_{1}-$ регулярньй элемент. 
Если же $y_{1} \notin \bar{V}_{2}$, то рассматриваем $\bar{V}_{2}=V_{2} \cap \bar{V}_{1}$, которое в силу нашего предположения строго содержится в $\bar{V}_{1}$.

Пусть уже построена цепочка $V=\bar{V}_{0} \supset \bar{V}_{1} \supset \cdots \supset \bar{V}_{i}$, где

$$
\bar{V}_{k}=\left\{\bar{v}_{k} \in L:\left(f_{k} \cdots f_{1} g\right)\left(\bar{v}_{k}\right) \neq 0\right\}, \quad k=1, \ldots, i,
$$

и $f_{k}=\tau_{r} \varphi_{y_{k-1}}^{-1}$ для некоторого элемента $y_{k-1} \in \bar{V}_{k-1}$, не принадлежащего $\bar{V}_{k}$.

Тогда, как при переходе от $\bar{V}_{1}$ к $\bar{V}_{2}$, строим $\bar{V}_{i+1}=V_{i+1} \cap \bar{V}_{i}$, где

$$
V_{i+1}=\left\{v_{i+1} \in L: f_{i+1}\left(v_{i+1}\right) \neq 0\right\}, \quad f_{i+1}=\tau_{r} \varphi_{y_{i}}^{-1}
$$

если для выбранного $y_{i} \in \bar{V}_{i}$ не выполняется условие принадлежности к $V_{i+1}$.

Предположим, что существует бесконечная строго убывающая цепочка открытых множеств

$$
\bar{V}_{0} \supset \bar{V}_{1} \supset \cdots \supset \bar{V}_{i} \supset \cdots, \quad \bar{V}_{i} \subset K^{n}
$$

Рассмотрим замкнутые подмножества

$$
W_{i}=\left\{w_{i} \in K^{n+1}:\left(f_{i} \cdots f_{1} g Y-1\right) w_{i}=0\right\} .
$$

Существует биекция $\pi_{i}$ между точками множеств $W_{i}$ и $\bar{V}_{i}$, которая является проекцией: $\pi_{i}\left(w_{1}^{i}, \ldots\right.$, $\left.w_{n}^{i}, w_{n+1}^{i}\right)=\left(w_{1}^{i}, \ldots, w_{n}^{i}\right)$, a $w_{n+1}^{i}=1 / \psi_{i}\left(w_{1}^{i}, \ldots, w_{n}^{i}\right), \psi_{i}=f_{i} \cdots f_{1} g,\left(w_{1}^{i}, \ldots, w_{n+1}^{i}\right) \in W_{i}$, $\left(w_{1}^{i}, \ldots, w_{n}^{i}\right) \in \bar{V}_{i}$. Но существование строго убывающей цепочки замкнутых множеств в $K^{n+1}$ противоречит нётеровости кольца многочленов $K\left[x_{1}, \ldots, x_{n}, Y\right]$. Следовательно, существует $i$ такое, что $W_{i}=W_{i+1}$, а поэтому и $\bar{V}_{i}=\bar{V}_{i+1}$. Но $\bar{V}_{i+1}=V_{i+1} \cap \bar{V}_{i}$, т.е. $\bar{V}_{i} \subset V_{i+1}=\left\{v_{i+1}\right.$ : $\left.\tau_{r} \varphi_{y_{i}}^{-1}\left(v_{i+1}\right) \neq 0\right\}$. По построению $y_{i} \in \bar{V}_{i}$ и $y_{i}=\varphi_{y_{i}} h_{i}$. В силу этих условий имеем $\tau_{r}\left(h_{i}\right)=$ $\tau_{r} \varphi_{y_{i}}^{-1}\left(y_{i}\right) \neq 0$. Итак, $h_{i}$ - регулярный элемент, принадлежащий картановской подалгебре $H \mathrm{c}$ энгелевым разложением. Как было отмечено выше, это доказывает пункты 1 и 2.

Пусть теперь $H=C(h), H^{\prime}=C\left(h^{\prime}\right)$ - две картановские подалгебры с энгелевым разложением, $\mathrm{a} h, h^{\prime}$ - соответствующие регулярные элементы. Пусть $e_{1}, \ldots, e_{r}, e_{r+1}, \ldots, e_{n} ; e_{1}^{\prime}, \ldots, e_{r}^{\prime}, e_{r+1}^{\prime}$, $\ldots, e_{n}^{\prime}$ - два базиса в $L$, согласованные с этими разложениями, причем $e_{1}, \ldots, e_{r}$ - базис $H, e_{1}^{\prime}, \ldots$, $e_{r}^{\prime}$ - базис $H^{\prime}$. Рассмотрим соответствующие полиномиальные отображения $P$ и $P^{\prime}$ в $L$. По ним определяются множества $U, U^{\prime}$ и $V, V^{\prime}$, когда $f=\tau_{r} P, f^{\prime}=\tau_{r} P^{\prime}$. В качестве $\bar{V}_{0}$ возьмем $V \cap V^{\prime}$. Тогда для любого $y_{0} \in \bar{V}_{0}$ определяем $x_{0}=P^{-1} y_{0}$ и $x_{0}^{\prime}=\left(P^{\prime}\right)^{-1} y_{0}$ и соответственно

$$
\varphi_{y_{0}}=\prod_{j=r+1}^{n}\left(1+x_{j}^{0} \text { ad } e_{j}\right) \quad \text { и } \quad \varphi_{y_{0}}^{\prime}=\prod_{j=r+1}^{n}\left(1+x^{\prime 0} \text { ad } e_{j}^{\prime}\right) .
$$

Затем определяем $f_{1}=\tau_{r} \varphi_{y_{0}}^{-1}$ и $f_{1}^{\prime}=\tau_{r}\left(\varphi_{y_{0}}^{\prime}\right)^{-1}$, а также

$$
V_{1}=\left\{v_{1} \in L: f_{1}\left(v_{1}\right) \neq 0\right\}, \quad V_{1}^{\prime}=\left\{v_{1}^{\prime} \in L: f_{1}^{\prime}\left(v_{1}^{\prime}\right) \neq 0\right\}
$$

Если $y_{0} \in V_{1} \cap V_{1}^{\prime}$, то $\tau_{r}\left(h_{0}\right) \neq 0, \tau_{r}\left(h_{0}^{\prime}\right) \neq 0$, где $h_{0}$ и $h_{0}^{\prime}$ - компоненты векторов $x_{0}$ и $x_{0}^{\prime}$, лежащие в $H$ и $H^{\prime}$, причем $y_{0}=\varphi_{y_{0}} h_{0}, y_{0}=\varphi_{y_{0}}^{\prime} h_{0}^{\prime}$. Тогда $h_{0}=\varphi_{y_{0}}^{-1} \varphi_{y_{0}}^{\prime} h_{0}^{\prime}$ и $\varphi_{y_{0}}^{-1} \varphi_{y_{0}}^{\prime}-$ отображение из формулировки теоремы.

В противном случае определяем $\bar{V}_{1}=V_{1} \cap V_{1}^{\prime} \cap \bar{V}_{0}$. Повторяя рассуждение из доказательства пунктов 1,2 , приходим к цепочке $\bar{V}_{0} \supset \bar{V}_{1} \supset \cdots \supset \bar{V}_{i} \supset \cdots$, где $\bar{V}_{i}=V_{i} \cap V_{i}^{\prime} \cap \bar{V}_{i-1}$, $f_{i}=\tau_{r} \varphi_{y_{i-1}}^{-1}, f_{i}^{\prime}=\tau_{r}\left(\varphi_{y_{i-1}}^{\prime}\right)^{-1}$ для некоторого $y_{i-1} \in \bar{V}_{i-1}$. Заканчивая как ранее, получаем, что существует $y_{i}$, для которого $y_{i}=\varphi_{y_{i}} h_{i}=\varphi_{y_{i}}^{\prime} h_{i}^{\prime}$, где $h_{i}, h_{i}^{\prime}$ - регулярные элементы, содержащиеся в картановских подалгебрах $H, H^{\prime}$. Тогда $h_{i}=\varphi_{y_{i}}^{-1} \varphi_{y_{i}}^{\prime} h_{i}^{\prime}$ и, как уже отмечалось при $i=0$, отображение $\varphi_{y_{i}}^{-1} \varphi_{y_{i}}^{\prime}$ удовлетворяет условию теоремы.

\section{СПИСОК ЦИТИРОВАННОЙ ЛИТЕРАТУРЫ}

1. Премет А. А. // Изв. АН. Сер. матем. 1986. Т. 50. №4. С. 788-800. 2. Премет А. А. // Матем. сб. 1989. Т. 180. № 4. С. 542-557. 3. Джекобсон Н. Алгебры Ли. М.: Мир, 1964. 4. Шафаревич И. Р. Основы алгебраической геометрии. М.: Наука, 1972. 